\title{
The clinical response to infliximab in rheumatoid arthritis is in part dependent on pretreatment tumour necrosis factor $\alpha$ expression in the synovium
}

\author{
C A Wijbrandts, ${ }^{1}$ M G W Dijkgraaf, ${ }^{2}$ M C Kraan, ${ }^{1}$ M Vinkenoog, ${ }^{1}$ T J Smeets, ${ }^{1}$ \\ H Dinant, ${ }^{1,3} \mathrm{~K} \mathrm{Vos}^{1,3}$ W F Lems, ${ }^{4,5} \mathrm{G}$ J Wolbink, ${ }^{1,5}$ D Sijpkens, ${ }^{1}$ B A C Dijkmans, ${ }^{3,4,5}$ \\ P P Tak'
}

${ }^{1}$ Division of Clinical Immunology and Rheumatology, Academic Medical Center/University of Amsterdam, Amsterdam, The Netherlands; ${ }^{2}$ Department of Clinical Epidemiology, Biostatistics and Bioinformatics, Academic Medical Center/ University of Amsterdam, Amsterdam, The Netherlands; ${ }^{3}$ Jan van Breemen Institute, Amsterdam, The Netherlands;

${ }^{4}$ Department of Rheumatology, Slotervaart Hospital,

Amsterdam, The Netherlands;

${ }^{5}$ VU Medical Center,

Amsterdam, The Netherlands

Correspondence to: Professor P P Tak, Division of Clinical Immunology and

Rheumatology, F4-218,

Academic Medical Center/

University of Amsterdam,

Meibergdreef 91105 AZ

Amsterdam, The Netherlands;

P.P.Tak@amc.uva.n

Accepted 18 November 2007 Published Online First

29 November 2007

\section{ABSTRACT}

Objective: To determine whether the heterogeneous clinical response to tumour necrosis factor (TNF) $\alpha$ blocking therapy in rheumatoid arthritis (RA) can be predicted by TNF $\alpha$ expression in the synovium before initiation of treatment.

Methods: Prior to initiation of infliximab treatment, arthroscopic synovial tissue biopsies were obtained from 143 patients with active RA. At week 16, clinical response was evaluated using the 28-joint Disease Activity Score (DAS28). Immunohistochemistry was used to analyse the cell infiltrate as well as the expression of various cytokines, adhesion molecules and growth factors. Stained sections were evaluated by digital image analysis. Student $t$ tests were used to compare responders (decrease in DAS28 $\geqslant 1.2$ ) with nonresponders (decrease in DAS28 <1.2) and multivariable regression was used to identify the independent predictors of clinical response.

Results: Synovial tissue analysis confirmed our hypothesis that the baseline level of TNF $\alpha$ expression is a significant predictor of response to TNF $\alpha$ blocking therapy. TNF $\alpha$ expression in the intimal lining layer and synovial sublining were significantly higher in responders than in non-responders $(p=0.047$ and $p=0.008$, respectively). The numbers of macrophages, macrophage subsets and T cells (all able to produce TNF $\alpha$ ) were also significantly higher in responders than in non-responders. The expression of interleukin (IL)1 $\beta$, IL6, IL18, IL10, E-selectin, intercellular adhesion molecule (ICAM)-1, vascular cell adhesion molecule (VCAM)-1, vascular endothelial growth factor (VEGF) and basic fibroblast growth factor (bFGF) was not associated with response to anti-TNF $\alpha$ treatment.

Conclusion: The effects of TNF $\alpha$ blockade are in part dependent on synovial TNF $\alpha$ expression and infiltration by TNF $\alpha$ producing inflammatory cells. Clinical response cannot be predicted completely, indicating involvement of other as yet unknown mechanisms.

Tumour necrosis factor $\alpha$ (TNF $\alpha$ ) blocking agents as treatment for rheumatoid arthritis (RA) were developed based on evidence that the pro-inflammatory cytokine TNF $\alpha$ plays an important role in the pathogenesis. ${ }^{1}$ Some patients however do not clinically respond to TNF $\alpha$ blockade. At present no factors have been identified that fully explain or predict the differential response.

One explanation for the heterogeneous clinical response may be found in the baseline variability in $\mathrm{TNF} \alpha$ expression among individual patients. ${ }^{23}$
Genetic studies have suggested that individuals predisposed to high TNF production could show worse responses to anti-TNF $\alpha$ therapy. ${ }^{45}$ By contrast, a recent study using an in vitro bioassay suggested that good responsiveness to anti-TNF therapy is associated with significantly higher TNF $\alpha$ bioactivity at baseline compared to nonresponding patients. ${ }^{6}$ Taken together, it remains to be determined which baseline cytokine profile distinguishes responding from non-responding patients in vivo. Another explanation for the diversity in response may be that inflammatory mediators other than TNF $\alpha$ drive different pathogenetic subsets of RA.

We hypothesised that the pretreatment TNF $\alpha$ level in the synovium might be related to clinical efficacy, where TNF $\alpha$ blocking therapy could be most effective in patients with high pretreatment $\mathrm{TNF} \alpha$ levels, as previously suggested in a small pilot study. ${ }^{7}$ In a prospective study we obtained arthroscopic synovial tissue samples from 143 patients with RA prior to initiation of infliximab therapy. We examined the cell infiltrate as well as the expression of cytokines, adhesion molecules and growth factors to identify predictors of clinical response.

\section{PATIENTS AND METHODS}

\section{Patients}

Consecutive patients with RA according to the American College of Rheumatology (ACR) criteria were enrolled in the study. All failed at least two disease-modifying antirheumatic drugs (DMARDs) including methotrexate (MTX) and had a 28-joint Disease Activity Score (DAS28) of $\geqslant 3.2$ when included in the study. Patients were on stable maximal tolerable MTX treatment (5-30 mg/ week). Oral corticosteroids ( $\leqslant 10 \mathrm{mg} /$ day) and non-steroidal anti-inflammatory drug (NSAIDs) were allowed if stable for at least 1 month prior to baseline. Concomitant medication was kept stable throughout the study. Previous use of a TNF blocking agent was an exclusion criterion. The Medical Ethics Committee of the Academic Medical Center, University of Amsterdam approved the protocol. All patients gave written informed consent.

\section{Treatment and evaluation of clinical response}

All patients were treated with infliximab according to the label for RA in a dosage of $3 \mathrm{mg} / \mathrm{kg}$ 
intravenously at baseline, week 2, week 6 and subsequently every 8 weeks. The DAS28 was evaluated at baseline and weeks 4, 8, 12 and 16 by specially trained research nurses.

For the analysis the absolute change in DAS28 ( $\triangle \mathrm{DAS} 28)$ at week 16 was dichotomised and defined as non-response $(\Delta \mathrm{DAS} 28<1.2)$ vs response $(\Delta \mathrm{DAS} 28 \geqslant 1.2)$. The dichotomy of the $\triangle \mathrm{DAS} 28$ (on average comparable with a $20 \%$ improvement in DAS28) was chosen because it is applied in daily clinical practice and required for prolongation of reimbursement for TNF $\alpha$ blocking therapy by insurance companies in The Netherlands. Response was evaluated at 16 weeks because a significant improvement is expected to occur within 3 to 4 months, after which alternative treatment should be considered. $^{8}$

\section{Arthroscopy and synovial biopsy}

Before the first infliximab infusion patients underwent a miniarthroscopy under local anaesthesia to obtain synovial tissue samples from an actively inflamed knee, ankle, wrist or metacarpophalangeal joint. ${ }^{9}$ Biopsies were taken with $2 \mathrm{~mm}$ forceps (Storz, Tuttlingen, Germany) from six or more sites within the joint to minimise sampling error. Biopsies were immediately snap frozen en bloc in Tissue Tek OCT (Miles, Elkhart, Indiana, USA) after collection. Sections of $5 \mu \mathrm{m}$ were cut in a cryostat and mounted on Star Frost adhesive glass slides (Knittelgläser, Braunschweig, Germany). Slides were stored at $-80^{\circ} \mathrm{C}$ until immunohistochemical staining.

\section{Immunohistochemical analysis}

Synovial sections were stained using the following monoclonal antibodies to analyse the infiltrate: anti-CD55 (67:Serotec, Oxford, UK) to detect fibroblast-like synoviocytes (FLS), antiCD68 (EBM11: DAKO, Glostrup, Denmark) to detect macrophages, anti-CD3 (SK7, Becton Dickinson (BD), California, USA) for $\mathrm{T}$ cells, anti-CD22 (CLB-B-ly/1,6B11, The Netherlands) for B cells and anti-CD38 (HB7, BD) for plasma cells. CD163 (Ber-MAC3; DAKO) was stained to detect resident tissue macrophages; infiltrating macrophages were evaluated by detection of myeloid related protein (MRP)8 (8-5c2, BMA Biomedicals, Augst, Switzerland) and MRP14 (S36.48, BMA Biomedicals). For the detection of cytokines, adhesion molecules and growth factors we used anti-human TNF $\alpha$ (52B83; Monosan, Brussels, Belgium), anti-interleukin (IL)6 (Nephrology Department, Leiden University Medical Center, Leiden, The Netherlands), anti-IL10 (23738.111, R\&D, Abingdon, UK), anti-IL18 (2d3b6, MD Biosciences, Minnesota, USA), anti-IL1 $\beta$ (2D8, ImmunoKontact, Oxford, UK), antiintercellular adhesion molecule (ICAM)-1 (MEM111, Sanbio, Erembodegem, Belgium), anti-vascular cell adhesion molecule (VCAM) (1G11B1, Sanbio), anti-E-selectin (BBIG-E4, R\&D), anti- vascular endothelial growth factor (VEGF) (N.010313 C-1, Santa Cruz, California, USA) and anti-basic fibroblast growth factor (bFGF) (F14220, BD). Staining of cellular markers was performed using a three-step immunoperoxidase method. ${ }^{2}$ For staining of cytokines, adhesion molecules and growth factors, biotinylated tyramine was used as amplification. For control sections the primary antibody was omitted or irrelevant immunoglobulins were applied.

\section{Digital image analysis}

All sections were analysed at random by trained analysts blinded for clinical outcome. Images were acquired and analysed by computer-assisted image analysis using a Syndia algorithm on a Qwin-based analysis system (Leica, Cambridge, UK) ${ }^{10} \mathrm{~A}$ total of 18 high-power fields per marker were analysed. Cellular markers were expressed as positive cells $/ \mathrm{mm}^{2}$ (counts $/ \mathrm{mm}^{2}$ ). Staining of cytokines, adhesion molecules and growth factors was expressed as integrated optical density $/ \mathrm{mm}^{2}\left(\mathrm{IOD} / \mathrm{mm}^{2}\right)$. CD68+ macrophages and TNF $\alpha$ expression were analysed separately in the intimal lining layer and the sublining.

\section{Statistical analysis}

Independent Student $t$ tests or Mann-Whitney $U$ tests were used to detect significant differences in baseline parameters between responders and non-responders. The $\chi^{2}$ test was employed to compare the percentage of erosive, IgM-rheumatoid factor (RF) and anti-cyclic citrullinated peptide (CCP) positive patients in the responder and non-responder groups. Variables with a $p$ value $<0.1$ in the univariable analysis were selected as possible predictors in a stepwise backward multivariable logistic regression model. Several exchangeable prediction sets were constructed based on the correlation structure among the potential predictors with strongly correlating predictors in different sets. The model with the highest explained variance according to Nagelkerke is reported. Correlations were assessed with the Pearson product-moment or Spearman rank-order correlation coefficients, whichever was appropriate. SPSS V.11.1.4 (Chicago, Illinois, USA) was used. Because the primary objective of this study was to find a pathogenetic mechanism in synovial tissue relating to clinical response, a per-protocol analysis was performed.

\section{RESULTS}

\section{Enrolment, discontinuation and exclusion}

A total of 143 patients were enrolled. The population was predominantly female (74\%), RF positive (71\%) with a mean (SD) age of 55 (13) years and mean disease duration of 125 (108) months. Erosions were present in 110 (77\%) patients. The mean DAS28 score was 5.9 (1.0) at baseline and the mean MTX dose was 18.2 (8.5) $\mathrm{mg} /$ week. In three patients, a flare of arthritis at week $6(n=1)$ and week $12(n=2)$ was the reason for discontinuation. These patients were analysed as nonresponders. Patients $(n=18)$ who violated the protocol or discontinued infliximab therapy (for reasons other than nonresponse) were excluded.

\section{Synovial tissue exclusion}

Following strict quality control, 23 synovial biopsies were excluded by a blinded assessor for absence of an intimal lining layer. After excluding 18 patients from the response analysis and 23 synovial biopsies from the tissue analysis a total of 103 completely evaluable patients remained for the analysis of synovial tissue in combination with clinical response.

\section{Baseline patient characteristics}

The remaining group of 103 patients who were analysed for response in combination with synovial tissue consisted of 71 females and 32 males, of whom $75 \%$ were rheumatoid factor positive. The mean (SD) age was 55 (13) years and the mean disease duration was 125 (110) months. Erosions were present in $79(77 \%)$ patients. The mean DAS28 score was $5.9(1.1)$ at baseline and the mean MTX dose was 18.8 (8.5) mg/week. Corticosteroids were used by $28(27 \%)$ patients with a mean dose of 8.0 (2.9) mg/day. On average, patients had failed treatment with 2.2 DMARDs before inclusion in the study. No notable changes occurred in the baseline characteristics of the 
Table 1 Baseline patient characteristics

\begin{tabular}{|c|c|c|c|c|}
\hline & $\begin{array}{l}\text { Analysed patients } \\
(n=103)\end{array}$ & Responders $(n=70)$ & $\begin{array}{l}\text { Non-responders } \\
(\mathrm{n}=\mathbf{3 3})\end{array}$ & p Value \\
\hline \multicolumn{5}{|l|}{ Demographics: } \\
\hline Age (years) & $55(13)$ & $54(13)$ & $56(12)$ & 0.40 \\
\hline Female (\%) & $71(69)$ & $51(73)$ & $20(61)$ & 0.21 \\
\hline \multicolumn{5}{|l|}{ Disease status: } \\
\hline $\begin{array}{l}\text { Disease duration } \\
\text { (months) }\end{array}$ & $125(110)$ & $123(111)$ & $130(110)$ & 0.80 \\
\hline Erosive disease (\%) & $79(77)$ & $57(81)$ & $22(67)$ & 0.10 \\
\hline RF positive (\%) & $77(75)$ & $57(81)$ & $20(61)$ & 0.02 \\
\hline Anti-CCP positive (\%) & $78(76)$ & $57(81)$ & $13(39)$ & 0.08 \\
\hline DAS28 & $5.9(1.1)$ & $6.0(1.0)$ & $5.6(1.2)$ & 0.07 \\
\hline $\begin{array}{l}\text { Patients global score } \\
(0-100 \mathrm{~mm})\end{array}$ & $60(22)$ & $62(21)$ & $56(23)$ & 0.23 \\
\hline ESR $(\mathrm{mm} / \mathrm{h})$ & $34(24)$ & $36(23)$ & $29(25)$ & 0.16 \\
\hline CRP (mg/dl) & $22(28)$ & $24(26)$ & $19(30)$ & 0.36 \\
\hline \multicolumn{5}{|l|}{ Drug treatments: } \\
\hline Previous DMARDs & $2.2(1.5)$ & $2.1(1.4)$ & $2.4(1.6)$ & 0.42 \\
\hline $\begin{array}{l}\text { Methotrexate (mg/ } \\
\text { week) }\end{array}$ & $18.8(8.5)$ & $19.5(8.2)$ & $17.1(8.9)$ & 0.18 \\
\hline $\begin{array}{l}\text { Receiving } \\
\text { corticosteroids (\%) }\end{array}$ & $28(27)$ & $22(31)$ & $6(18)$ & 0.22 \\
\hline Receiving NSAIDs (\%) & $52(50)$ & $35(50)$ & 17 (52) & 0.89 \\
\hline
\end{tabular}

analysed 103 patients compared to the baseline characteristics of all 143 enrolled patients (see table 1).

\section{Clinical response}

Of 103 patients, 70 (68\%) were DAS28 responders and $33(32 \%)$ non-responders at week 16 . The clinical efficacy matched the expected $60-70 \%$ responders observed in previous randomised controlled trials with infliximab in patients with RA. The mean change in DAS28 was 1.84 (1.26) at week 16. For nonresponders the average $\triangle \mathrm{DAS} 28$ was 0.45 (0.58) compared to $2.52(0.89)$ for responders. This difference was highly significant $(\mathrm{p}<0.001)$.

All baseline patient characteristics were also tested for differences between responders and non-responders. The DAS28 at baseline was nearly significant and was included in the prediction model $(p=0.07)$. The same holds for anti-CCP positivity $(p=0.08)$. Finally, there were significantly more rheumatoid factor positive patients in the responder compared to the non-responder group $(p=0.020)$, (see table 1$)$.

\section{Baseline synovial TNF $\alpha$ expression and the number of major TNF $\alpha$ producing cells are associated with clinical response ( $\triangle D A S 28 \geqslant 1.2)$ after 16 weeks of TNF $\alpha$ blocking therapy}

Scatter diagrams of the synovial predictors in individual patients in both response groups are presented in fig 1. TNF $\alpha$ expression levels were higher in the synovial sublining of responders compared to non-responders $(p=0.008)$ (fig 1A). Similarly, $\mathrm{TNF} \alpha$ expression in the intimal lining layer was higher in responders compared to non-responders $(p=0.047)$. Table 2 shows the associations of synovial markers and response.

Because macrophages are known to be the main TNF $\alpha$ producing cells in the synovium, the number of macrophages was studied. The mean number of CD68+ sublining macrophages was significantly higher in responders than in nonresponders $(p=0.029)$ (fig $1 C)$. Similarly, the number of CD163+ resident tissue macrophages as well as the number of infiltrating MRP8+ and MRP14+ macrophages was higher in responders $(p=0.017, p=0.018$ and $p=0.024$, respectively) (fig 1D). Finally, the median number of CD3+ $\mathrm{T}$ cells was higher in responders $(p=0.001)$ (fig $1 B)$.

\section{Baseline TNF $\alpha$ is the only significant synovial predictor of response}

The relationship between TNF $\alpha$ expression and clinical response was confirmed by stepwise backward multivariable logistic regression analysis. Due to multicolinearity among predictors, 10 exchangeable bivariate prediction sets were constructed consisting of (1) TNF $\alpha$ expression in either the intimal lining layer or synovial sublining with (2) one out of five cellular markers. This revealed that TNF $\alpha$ expression in the synovial sublining was the only independent, early determinant of therapy response $(p=0.011)$, explaining just about $10 \%$ of the variance in response to therapy $\left(R^{2}=0.099\right.$, according to Nagelkerke, odds ratio $(\mathrm{OR})=1.013)$. Likewise, TNF $\alpha$ expression in the intimal lining layer rather than synovial sublining explained $9 \%$ of the variance in response to therapy $(p=0.020)$, $\left(\mathrm{R}^{2}=0.089, \mathrm{OR}=1.017\right)$. Thus, after adjustment for $\mathrm{TNF} \alpha$ in a bivariate logistic regression model including either the numbers of CD68+ sublining macrophages, CD163+, MPR8+, MRP14+ macrophages, or CD3+ $\mathrm{T}$ cells, these cellular markers were no longer significantly associated with response. The removal of these cellular markers from the model is consistent with the notion that these cells are the main producers of TNF $\alpha$.

\section{Contribution of disease activity at baseline to the prediction of response}

To assess the value of a combined prediction model consisting of clinical and synovial data we added the relevant clinical variables (DAS28 score, the presence of IgM-RF or anti-CCP antibodies) to the 10 bivariate synovial models. With stepwise backward multivariate logistic regression this resulted in a significant prediction model with TNF $\alpha$ expression in the 
Figure 1 A. The median synovial sublining tumour necrosis factor (TNF) $\alpha$ expression was higher in responders compared to non-responders $(p=0.008)$. $B$. The median number of CD3+ T cells in responders vs non-responders $(p=0.001)$. (C) The mean number of CD68+ sublining macrophages was also higher in responders 576 (428), than in non-responders $387(338)(p=0.029)$.

(D) CD163+ macrophages in responders vs non-responders $(p=0.017)$.
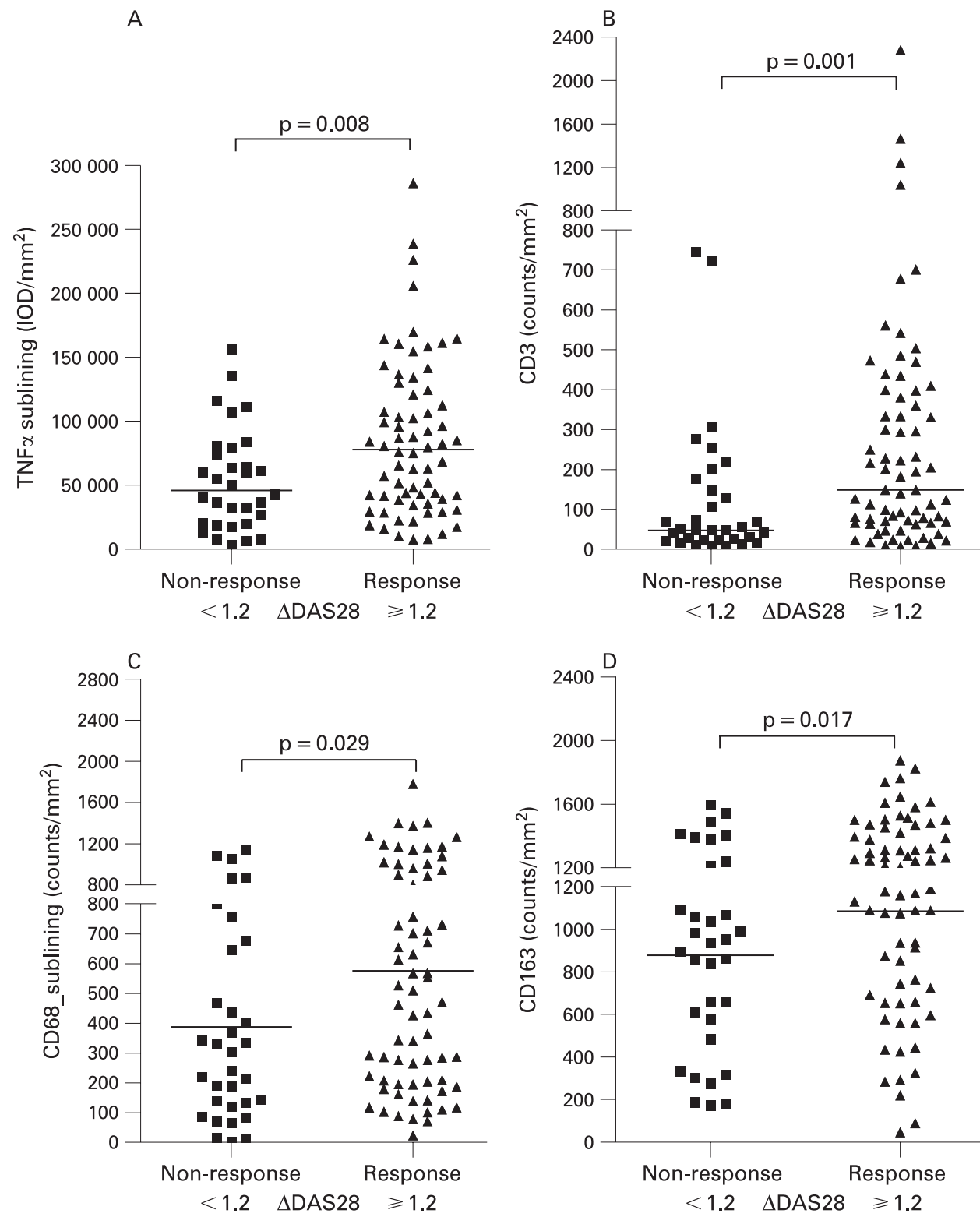

synovial sublining $(p=0.008, O R=1.014)$ and the DAS28 $(\mathrm{p}=0.031, \mathrm{OR}=1.611)$ with an increased explained variance of $17 \%\left(R^{2}=0.172\right)$. A similar analysis except with TNF $\alpha$ expression in the intimal lining layer $(p=0.023, O R=1.017)$ resulted in a significant model including the DAS28 $(p=0.044$, $\mathrm{OR}=1.588)$ and the presence of anti-CCP antibodies $(\mathrm{p}=0.046$, $\mathrm{OR}=3.038)\left(\mathrm{R}^{2}=0.182\right)$.

\section{Baseline IL1ß, IL6, IL18 and IL10 expression is not associated with response to TNF $\alpha$ blocking therapy}

In addition to TNFa we studied the expression of other cytokines such as IL18, which is believed to operate mainly upstream of TNF $\alpha$, while IL1 $\beta$ and IL6 appear to act mainly downstream of TNF $\alpha$. Despite the known interplay of these cytokines with TNF $\alpha$ we found no relationship between synovial expression levels of either of these cytokines and clinical response. We did find, however, a significant correlation between the level of IL1 $\beta$ expression and TNF $\alpha$ expression $(r=0.306, p=0.014)$. The expression level of IL10 was not different between response groups (see table 2).
Baseline expression of adhesion molecules and angiogenic factors is not associated with response to TNF $\alpha$ blocking therapy

In accordance with their function in leukocyte recruitment a significant correlation was found between the number of sublining macrophages on the one hand and the expression of the vascular adhesion molecules E-selectin (Spearman rho correlation coefficient $0.275-0.536, \mathrm{p}<0.01$ ) on the other. However, the level at which the adhesion molecules were expressed in the synovium at baseline did not differ between responders and non-responders. Furthermore, VEGF is known to mediate angiogenesis and is regulated by pro-inflammatory cytokines such as TNF $\alpha$. However, no association was found between VEGF expression and response (table 2). Similarly, no difference was found in the expression of bFGF.

\section{DISCUSSION}

The primary objective of this study was to investigate whether immunohistological assessment of the cell infiltrate and cytokine expression in the synovium prior to initiation of 
Table 2 Associations of studied synovial predictors with clinical response

\begin{tabular}{|c|c|c|c|}
\hline & \multicolumn{2}{|l|}{$\triangle D A S 28$ score } & \multirow[b]{2}{*}{ p Value } \\
\hline & Responders $(n=70) \geqslant 1.2$ & Non-responders $(\mathrm{n}=33)<1.2$ & \\
\hline \multicolumn{4}{|c|}{ Cytokines (IOD/mm²): } \\
\hline TNF $\alpha$ sublining & 77947 (38 710-123 535) & 46033 (19 890-78 129) & 0.008 \\
\hline IL1 $\beta$ & 56278 (31 063-81 655) & 54484 (32 098-129 041) & 0.814 \\
\hline IL6 & 50537 (41 939) & $38364(28527)$ & 0.135 \\
\hline \multicolumn{4}{|c|}{ Cellular markers (counts $/ \mathrm{mm}^{2}$ ) } \\
\hline CD55 & $652(418)$ & 694 (389) & 0.895 \\
\hline CD3 & $149(66-385)$ & $47(22-163)$ & 0.001 \\
\hline CD68 lining & $374(253)$ & $295(184)$ & 0.130 \\
\hline CD68 sublining & $576(428)$ & $387(338)$ & 0.029 \\
\hline CD163 & $1100(432)$ & $878(433)$ & 0.017 \\
\hline \multicolumn{4}{|c|}{ Adhesion molecules (IOD/mm²) } \\
\hline ICAM & 30790 (33 016) & $22321(21706)$ & 0.195 \\
\hline VCAM & $80065(52630)$ & 70701 (44 574) & 0.387 \\
\hline E-Selectin & 37651 (40 134) & 30488 (29 972) & 0.373 \\
\hline \multicolumn{4}{|c|}{ Growth factors (IOD/mm $\left.\mathrm{mm}^{2}\right)$} \\
\hline VEGF & 18252 (78 689) & $5776(5372)$ & 0.374 \\
\hline bFGF & $209(736)$ & $64(163)$ & 0.276 \\
\hline
\end{tabular}

*Data are presented as mean (SD) or median (interquartile range), whichever appropriate. $p$ Values $<0.05$ (two-sided) were considered significant.

bFGF, basic fibroblast growth factor; ICAM, intercellular adhesion molecule; IF, interferon; IOD, integrated optical density; MRP, myeloid related protein; TNF, tumour necrosis factor; VCAM, vascular cell adhesion molecule; VEGF, vascular endothelial growth factor.

TNF $\alpha$ blocking therapy could predict clinical response in patients with RA. We could confirm our hypothesis that the level of synovial TNF $\alpha$ expression is a significant early predictor of the response. This was shown by increased TNF $\alpha$ expression levels in the intimal lining layer and synovial sublining of responding compared to non-responding patients. In line with these findings, there was increased infiltration by macrophages, including CD163+ resident tissue macrophages and MRP8+ and MRP14+ infiltrating macrophages, as well as $\mathrm{T}$ cells in responders vs non-responders. It is important to note that these cells are the main source of TNF $\alpha$ in the synovium of patients with RA. ${ }^{11}$

Consistent with the clinical experience that the response to $\mathrm{TNF} \alpha$ blockade is not a dichotomous phenomenon, ${ }^{12}$ there was no distinct threshold value in TNF $\alpha$ expression in the synovium of patients with RA. Multivariate logistic regression analysis of synovial markers showed that TNF $\alpha$ expression in the sublining could explain about $10 \%$ of the variance in response to therapy. After adjusting for disease activity at baseline this further increased to $17 \%$. Hence, the predictive value of synovial TNF expression is statistically significant, but overall limited. This clearly indicates that variables other than synovial TNF $\alpha$ expression are involved as well. The results presented here show for the first time that the expression of IL1 $\beta$, IL6, IL18, IL10, E-selectin, ICAM-1, VCAM-1, VEGF and bFGF is not associated with clinical response to anti-TNF $\alpha$ treatment. Thus, the features of synovial inflammation at baseline in responders to anti-TNF therapy compared to non-responders do not merely represent a greater amount of inflammation, but the results presented here underscore the importance of specific inflammatory pathways. Future work should expand the search for other biomarkers and molecular networks to better understand the variable response to anti-TNF $\alpha$ therapy.
The search for predictors of response is important in the context of personalised medicine, which may be an effective approach to increase the percentage of patients exhibiting a robust response to a given treatment. Previous work has clearly suggested that RA consists of different pathogenetic subsets, leading to common signs and symptoms associated with what we at present define clinically as RA. ${ }^{2}{ }^{13}$ Thus, it is conceivable that, for instance, TNF $\alpha$ expression is more important in some patients than in others, and that TNF blockade could be more effective in the former. Although we are as yet not able to predict the response sufficiently to select patients who are likely to have a beneficial response to a specific treatment before initiation of therapy and to guide treatment decisions, the results of the present study do provide proof of principle that this might be achieved by further optimisation of the biomarkers or perhaps combinations with other clinical and biological variables that need to be identified. The relevance of this approach is underscored by the expanding array of biological therapies and their costs. ${ }^{14}$

There is no evidence that simple measurement of plasma TNF $\alpha$ levels can be used to predict clinical response to TNF $\alpha$ blockade. As it appears logical that patients producing high levels of TNF $\alpha$ at the site of inflammation are more likely to benefit from TNF $\alpha$ blockade than those with lower TNF $\alpha$ levels, we focused on the synovium as the primary target of RA. The association between pretreatment TNF $\alpha$ expression in the synovium and clinical response to $3 \mathrm{mg} / \mathrm{kg}$ of infliximab described here in 103 patients with RA confirms and extends a trend observed in a pilot study in patients with RA where 4 out of 8 patients who met the ACR50 response criteria at 2 weeks after initiation of infliximab $(10 \mathrm{mg} / \mathrm{kg})$ therapy were those with the highest levels of TNF $\alpha$ expression in the synovium, as shown by immunohistochemistry. ${ }^{7}$ Of interest, 
the findings are also in accordance with a recent study suggesting that clinical response at 52 weeks is associated with significantly higher circulating TNF $\alpha$ bioactivity (measured by an in vitro bioassay) at baseline in responding compared to nonresponding patients. ${ }^{6}$ Together, these studies support the notion that the initial clinical response to TNF $\alpha$ blockade is related to pretreatment levels of $\mathrm{TNF} \alpha$ production. In patients who initially respond, but loose response over time, other mechanisms such as formation of antibodies against the drug may be operative. ${ }^{15}$

In conclusion, the results presented here show proof of principle that the heterogeneous response to TNF $\alpha$ blockade is associated with TNF $\alpha$ expression in the inflamed synovium. Future work should expand the search for other biomarkers and molecular networks as well as combinations with clinical variables. Thus, the prediction of how a patient will respond might come in reach of the treating doctor.

Acknowledgements: The authors wish to thank Professor A H Zwinderman, head of the department of Department of Clinical Epidemiology, Biostatistics and Bioinformatics at the Academic Medical Center/University of Amsterdam, for reviewing the statistical analysis. We also wish to thank Desiree Pots for assisting with the immunohistochemical staining, and research nurses Nitolanda van Rijn and Natasja Cassin for performing clinical assessments.

Funding: This study was funded by a Health Care Efficiency Research Programme grant from The Netherlands Organization for Health Research and Development (ZonMw) in assignment of The Netherlands Organization for Scientific Research (NWO) (grant number 945-02-029), the Dutch Arthritis Association and the European Community's FP6 funding (Autocure).

Competing interests: PPT has served as a consultant for Abbott, Amgen, Centocor and Wyeth.

Ethics approval: The Medical Ethics Committee of the Academic Medical Center, University of Amsterdam approved the protocol. All patients gave written informed consent.

This publication reflects only the authors' views. The European Community is not liable for any use that may be made of the information herein.

\section{REFERENCES}

1. Feldmann M, Brennan FM, Maini RN. Role of cytokines in rheumatoid arthritis. Annu Rev Immunol 1996;14:397-440.

2. Tak PP, Smeets TJ, Daha MR, Kluin PM, Meijers KA, Brand R, et al. Analysis of the synovial cell infiltrate in early rheumatoid synovial tissue in relation to local disease activity. Arthritis Rheum 1997;40:217-25.

3. Ulfgren AK, Grondal L, Lindblad S, Khademi M, Johnell O, Klareskog L, et al. Interindividual and intra-articular variation of proinflammatory cytokines in patients with rheumatoid arthritis: potential implications for treatment. Ann Rheum Dis 2000;59:439-47.

4. de Vries N, Tak PP. The response to anti-TNF- $\alpha$ treatment: gene regulation at the bedside. Rheumatology (Oxford) 2005;44:705-7.

5. Kang CP, Lee KW, Yoo DH, Kang C, Bae SC. The influence of a polymorphism at position -857 of the tumour necrosis factor $\alpha$ gene on clinical response to etanercept therapy in rheumatoid arthritis. Rheumatology (Oxford) 2005; 44:547-52.

6. Marotte H, Maslinski W, Miossec P. Circulating tumour necrosis factor- $\alpha$ bioactivity in rheumatoid arthritis patients treated with infliximab: link to clinical response. Arthritis Res Ther 2005; 7:R149-55.

7. Ulfgren AK, Andersson U, Engstrom M, Klareskog L, Maini RN, Taylor PC. Systemic anti-tumor necrosis factor $\alpha$ therapy in rheumatoid arthritis down-regulates synovial tumor necrosis factor $\alpha$ synthesis. Arthritis Rheum 2000;43:2391-6.

8. Furst DE, Breedveld FC, Kalden JR, Smolen JS, Burmester GR, Dougados M, et al. Updated consensus statement on biological agents for the treatment of rheumatoid arthritis and other immune mediated inflammatory diseases (May 2003). Ann Rheum Dis 2003;62(Suppl 2):ii2-ii9.

9. Kraan MC, Reece RJ, Smeets TJ, Veale DJ, Emery P, Tak PP. Comparison of synovial tissues from the knee joints and the small joints of rheumatoid arthritis patients: Implications for pathogenesis and evaluation of treatment. Arthritis Rheum 2002:46:2034-8.

10. Haringman JJ, Vinkenoog M, Gerlag DM, Smeets TJ, Zwinderman AH, Tak PP. Reliability of computerized image analysis for the evaluation of serial synovial biopsies in randomized controlled trials in rheumatoid arthritis. Arthritis Res Ther 2005;7:R862-7.

11. Bazzoni F, Beutler B. The tumor necrosis factor ligand and receptor families. N Engl J Med 1996;334:1717-25.

12. Van Vollenhoven RF, Klareskog L. Clinical responses to tumor necrosis factor $\alpha$ antagonists do not show a bimodal distribution: data from the Stockholm tumor necrosis factor $\alpha$ followup registry. Arthritis Rheum 2003;48:1500-3.

13. van der Pouw Kraan TC, van Gaalen FA, Kasperkovitz PV, Verbeet NL, Smeets TJ Kraan MC, et al. Rheumatoid arthritis is a heterogeneous disease: evidence for differences in the activation of the STAT-1 pathway between rheumatoid tissues. Arthritis Rheum 2003;48:2132-45.

14. Smolen JS, Aletaha D. Challenges of predicting treatment response in patients with rheumatoid arthritis. Nat Clin Pract Rheumatol 2005;1:62-3.

15. Wolbink GJ, Vis M, Lems WF, Voskuyl AE, de Groot E, Nurmohamed MT, et al. Development of antiinfliximab antibodies and relationship to clinical response in patients with rheumatoid arthritis. Arthritis Rheum 2006;54:711-15. 